\title{
Comparison of univariate and multivariate linkage analysis of traits related to hypertension

\author{
Courtney Gray-McGuire ${ }^{1,2}$, Yeunjoo Song ${ }^{1}$, Nathan J Morris ${ }^{1}$ \\ and Catherine M Stein* ${ }^{1}$
}

\author{
Addresses: ${ }^{1}$ Department of Epidemiology and Biostatistics, Case Western Reserve University, 2103 Cornell Road, Cleveland, Ohio 44106, USA \\ and ${ }^{2}$ Oklahoma Medical Research Foundation, 121 North Shartel Avenue, Oklahoma City, Oklahoma 73102, USA \\ E-mail: Courtney Gray-McGuire - Courtney-Gray-McGuire@omrf.org; Yeunjoo Song - song@darwin.cwru.edu; \\ Nathan J Morris - Nathan.morris@case.edu; Catherine M Stein* - catherine.stein@case.edu \\ *Corresponding author
}

from Genetic Analysis Workshop 16

St Louis, MO, USA 17-20 September 2009

Published: 15 December 2009

BMC Proceedings 2009, 3(Suppl 7):S99 doi: 10.1186/1753-6561-3-S7-S99

This article is available from: http://www.biomedcentral.com/1753-656I/3/S7/S99

(C) 2009 Gray-McGuire et al; licensee BioMed Central Ltd.

This is an open access article distributed under the terms of the Creative Commons Attribution License (http://creativecommons.org/licenses/by/2.0), which permits unrestricted use, distribution, and reproduction in any medium, provided the original work is properly cited.

\begin{abstract}
Complex traits are often manifested by multiple correlated traits. One example of this is hypertension (HTN), which is measured on a continuous scale by systolic blood pressure (SBP). Predisposition to HTN is predicted by hyperlipidemia, characterized by elevated triglycerides (TG), low-density lipids (LDL), and high-density lipids (HDL). We hypothesized that the multivariate analysis of TG, LDL, and HDL would be more powerful for detecting HTN genes via linkage analysis compared with univariate analysis of SBP. We conducted linkage analysis of four chromosomal regions known to contain genes associated with HTN using SBP as a measure of HTN in univariate Haseman-Elston regression and using the correlated traits TG, LDL, and HDL in multivariate Haseman-Elston regression. All analyses were conducted using the Framingham Heart Study data. We found that multivariate linkage analysis was better able to detect chromosomal regions in which the angiotensinogen, angiotensin receptor, guanine nucleotide-binding protein 3 , and prostaglandin 12 synthase genes reside. Univariate linkage analysis only detected the AGT gene. We conclude that multivariate analysis is appropriate for the analysis of multiple correlated phenotypes, and our findings suggest that it may yield new linkage signals undetected by univariate analysis.
\end{abstract}

\section{Background}

Many common diseases are characterized by several correlated factors. These may be the results of a battery of test scores or they may be series of serum lipid levels or anthropometric measures. It is likely that these correlated traits are influenced by common genes (pleiotropy) or at least genes in common pathways. Eaves et al. [1] point out that the covariance induced on a set of phenotypes segregating at one locus may differ from that induced by segregation at another locus, making the interpretation of univariate results quite difficult [2]. Additionally, the use of multivariate approaches can 
increase the power and precision of linkage estimates $[3,4]$ and can serve as a mechanism by which to control for multiple comparisons when there are several traits of interest [5].

Hypertension (HTN), defined by consistent, elevated blood pressure (systolic (SBP) and/or diastolic (DBP)) is an example of a multifactorial trait correlated with multiple other phenotypes. Certainly, environmental factors such as diet and exercise are important determinants of HTN, but the influence of genetic factors is also well supported. In fact, there are a small percentage of HTN cases with monogenic forms of the disease [6]. The results of several linkage and association studies of HTN and SBP have suggested candidate genes, including: angiotensinogen (AGT) [7], tumor necrosis factor receptor-2 (TNFR2) [8], endothelin-converting enzyme-1 (ECE1) [9], angiotensin receptor (AGTR1), beta-3 subunit of guanine nucleotide-binding protein (GNB3) [10], and prostaglandin I2 synthase (PTGIS) [11].

This study compares a univariate and multivariate method for linkage analysis using a measure HTN, specifically SBP, and then a set of correlated phenotypes influencing SBP as examples and using the location of established candidate genes as our metric. It is our contention that by using information from multiple factors correlated with SBP levels and each other (rather than either the single continuous or dichotomous trait), we will be more effective in identifying regions of the genome previously demonstrated to be linked to SBP levels without as great a penalty for multiple testing.

\section{Methods}

\section{Phenotype data}

We analyzed the Framingham Heart Study data including observations for Original, Offspring, and Generation 3 cohorts as long as data for all the traits of interest were present. Data were obtained and used in compliance with the data use agreement and Case Western Reserve University Institutional Review Board approval. Lowdensity lipoprotein (LDL) values were derived using high-density lipoprotein (HDL) and total cholesterol values as required by the Friedewald equation. We used data from the last visit for the Original and Offspring cohorts where all variables of interest were measured. There was only one observation available for the Generation 3 cohort, so that is what we used. The choice of using the latest time point was made in an effort to obtain the most extreme values in our phenotypes of interest (because the study participants would be older).

Based on preliminary model-fitting statistics, we adjusted for age at exam, sex, and the interaction of age at exam by sex by including them as covariates in all analyses. We adjusted for possible HTN treatment by adding a constant of 10 to SBP [12]. Finally, we applied a natural log transformation to triglyceride (TG), HDL, and LDL before analysis to best approximate normality. Within-individual and sibling pair correlations across traits were estimated using FCOR (S.A.G.E. v5.4.1).

\section{Marker data}

Because the purpose of our study is to demonstrate the utility of a multivariate linkage method, using the full set of $500 \mathrm{k}$ genome-wide single-nucleotide polymorphisms (SNPs) would lead to far too much redundancy in the data (due to linkage disequilibrium). Therefore, we selected markers every $1000 \mathrm{~kb}$ (i.e., approximately every centimorgan) on which to perform linkage analysis. We further reduced the size of the dataset of analyses by choosing only chromosomes on which there were both previously published linkage signals and candidate genes, including chromosomes $1,3,12$, and 20, containing candidate genes AGT (204 cM), TNFR2 (13 cM), and ECE1 (21.5 cM); AGTR1 (150 cM); GNB3 (6.8 cM); and PTGIS (47.5 cM), respectively. Our final marker list comprised 611 SNPs.

\section{Linkage analysis}

Prior to linkage analysis, mendelian inconsistencies were identified in the data using MARKERINFO (S.A.G.E. v5.4.1) and the genotypes of all individuals in a family with an inconsistency were set to missing for the given marker. GENIBD was used to estimate the proportion of alleles shared identically by descent (IBD) between sibling pairs using information from individual and neighboring markers (i.e., multipoint). Parental genotypes from the original cohort were used where available in the estimation of IBD sharing. Four pedigrees with complex structure and more than 200 members were removed before IBD sharing estimation.

The univariate phenotype of interest was the quantitative trait SBP, a measure of hypertension. The multivariate traits comprised three phenotypes highly correlated with SBP: TG, HDL, and LDL (Table 1). Thus, we used univariate linkage analysis to analyze SBP and multivariate linkage to analyze TG, HDL, and LDL jointly.

\section{Univariate linkage}

We used performed Haseman-Elston regression [13] on the transformed SBP levels. As implemented in SIBPAL, the Haseman-Elston method regresses a weighted combination of the squared trait difference and squared mean-corrected trait sum on the estimated proportion of alleles shared IBD to account for the non-independence 
Table I: Within-individual and sibling-sibling pair correlations for TG, HDL, LDL, and SBP ${ }^{a}$

\begin{tabular}{lcccc}
\hline & SBP & TG & HDL & LDL \\
\hline SBP & $\mathbf{0 . 2 4 1 7 ^ { \mathbf { b } }}$ & $\mathbf{0 . 0 8 2 1 ^ { \mathbf { b } }}$ & $\mathbf{- 0 . 0 2 8 3}$ & $\mathbf{- 0 . 0 2 9 2}$ \\
TG & $0.2726^{\mathrm{b}}$ & $\mathbf{0 . 1 9 8 7}^{\mathrm{b}}$ & $\mathbf{- 0 . 1 2 2 2 ^ { \mathrm { b } }}$ & $\mathbf{0 . 0 4 4 0 ^ { \mathbf { c } }}$ \\
HDL & $-0.1293^{\mathrm{b}}$ & $-0.5124^{\mathrm{b}}$ & $\mathbf{0 . 2 0 9 1 ^ { \mathrm { b } }}$ & $\mathbf{0 . 0 1 5 3}$ \\
LDL & $0.1464^{\mathrm{b}}$ & $0.1684^{\mathrm{b}}$ & $\mathbf{- 0 . 1 2 4 6 ^ { \mathrm { b } }}$ & $\mathbf{0 . 1 9 7 9 ^ { \mathbf { a } }}$ \\
\hline
\end{tabular}

${ }^{a}$ Within-individual cross-trait correlations are shown in the lower triangle of the table (unbolded font); sibpair cross-trait correlations are in bold font. Correlations bolded on the diagonal are sibpair correlations within trait.

bIndicates significance at $p<0.000 \mathrm{I}$.

CIndicates significance at $p<0.01$.

of the sums and differences, as well as the nonindependence of sibling-pairs (option W4). Our final sample comprised 3985 full and half-sibling pairs.

\section{Multivariate linkage}

To conduct multivariate linkage, we used the new S.A.G.E. program RELPAL, which implements a test similar to the multivariate Haseman-Elston [14]. This model is built on the two-level Haseman-Elston [15], which incorporates individual-level covariates at the first level, and performs linkage analysis of multiple traits at the second level. A one-sided score test was used which is asymptotically equivalent to the likelihood-ratio test [16]. Because this method uses a robust sandwich-type estimator, it should maintain correct type I error asymptotically even when the data do not follow a multivariate normal distribution. Significance levels were determined using a novel algorithm described elsewhere [17]. This method has an advantage over other multivariate methods because it retains the power associated with variance-components models while still being robust to normality assumptions. Our final sample comprised 3940 full and half-sibling pairs. The reduction from the 3985 in our univariate analysis was due to the requirement of complete data for all three traits of interest.

\section{Results}

\section{Correlations}

Cross-trait correlations, both within individual and sibling pair, are shown in Table 1. All within-individual cross-trait correlations were significant at $p<0.0001$ (Table 1), demonstrating two things: first, that TG, HDL, and LDL are indeed adequate surrogates for SBP, and second, that the shared variance between these two traits implies possible pleiotropic effects. Unlike the withinindividual correlations, the sibling correlations for SBP with HDL and LDL were not significant. However, TG, HDL, and LDL in one sibling were all significantly correlated with TG, HDL, and LDL in the other sib at $p<0.01$. The latter result supports the usefulness of joint analysis of these traits (i.e., identification of common genetic determinants), while the former gives credence to the multivariate analysis because the subphenotypes appear to co-vary within a family more than does the univariate trait SBP.

\section{Linkage analysis}

Of the four chromosomal regions analyzed, we found a few regions of note linked to SBP using the univariate analysis at the $\alpha=0.01$ level. These regions were on chromosome 1 between 159 and $172 \mathrm{cM}$, at $186 \mathrm{cM}$, and between 195 and $198 \mathrm{cM}$ (Figure 1). These results are within 6 to $32 \mathrm{cM}$ of the AGT gene $(204 \mathrm{cM})$ and therefore could be representative of this effect [18], but are certainly not precise enough to rule out the effect of other genes in these regions.

Using the multivariate model, we detected two regions significant at $\alpha=0.01$. On chromosome 1 (Figure 1A) we detected a region between 198 and $209 \mathrm{cM}$ and also at the first SNP. The AGT gene is contained within this first interval $(204 \mathrm{cM})$ and TNFR2 is located at $13 \mathrm{cM}$, very near to the first SNP analyzed. On chromosome 12 (Figure 1C), we observed linkage between 18 and $35 \mathrm{cM}$; again, within $9 \mathrm{cM}$ of the previously associated GNB gene. At the $\alpha=0.05$ level, we observed linkage on chromosome 3 (Figure 1B) between 158 and $171 \mathrm{cM}, 8$ cM from AGTR1.

\section{Discussion}

In this study, our objective was to compare univariate and multivariate linkage results of four chromosomal regions known to contain mendelian genes linked to HTN. Linkage analysis remains a relevant approach for the analysis of rare and/or mendelian genetic effects $[19,20]$, as well as for providing a priori weights for association analysis [21], so we examined a newly implemented and truly multivariate linkage analysis model. We recognize that the most compelling demonstration of new methodology is via simulated data. However, the effect sizes represented in the real data were much more suited to linkage analysis. And, because there were established effects to which we could compare our results, this data represented a reasonable alternative. Indeed, we used a univariate and multivariate linkage approach to analyze SBP or traits related to HTN (and highly correlated with SBP) - TG, LDL, and HDL, respectively. We examined the same regions known to contain genes predisposing to risk of HTN. Our multivariate linkage analysis identified more nominally significant regions, and these results covered the chromosomal regions where the AGT, TNFR2, AGTR, and GNB3 genes reside. Though the univariate results were 
A

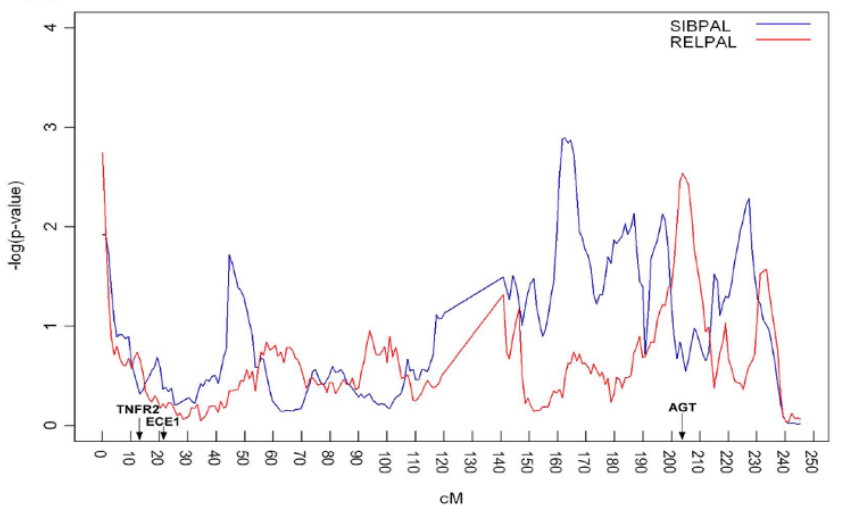

C

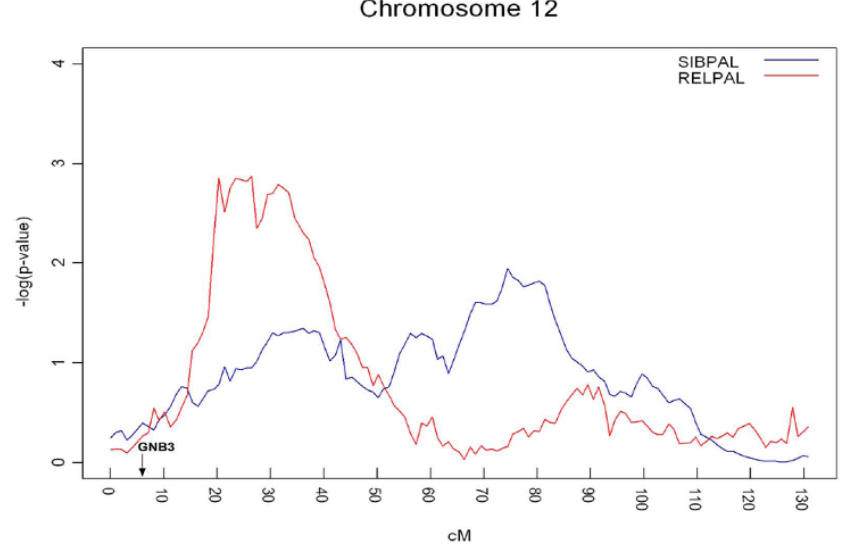

B

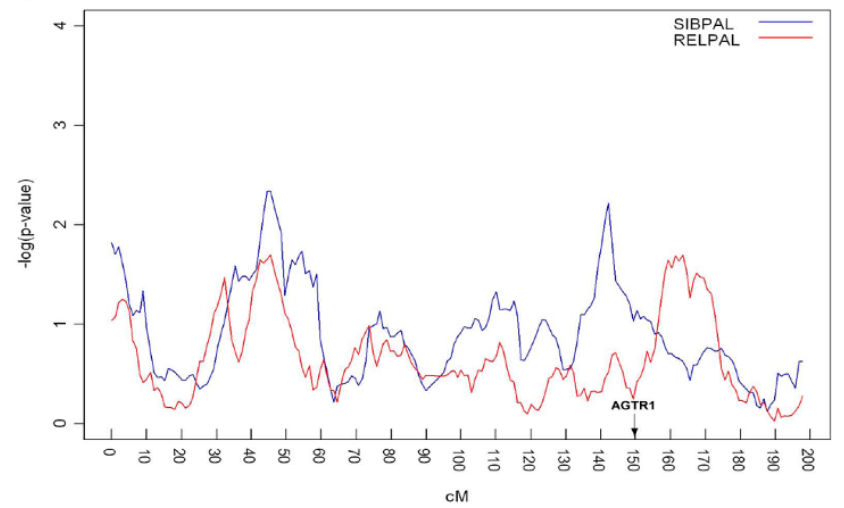

D

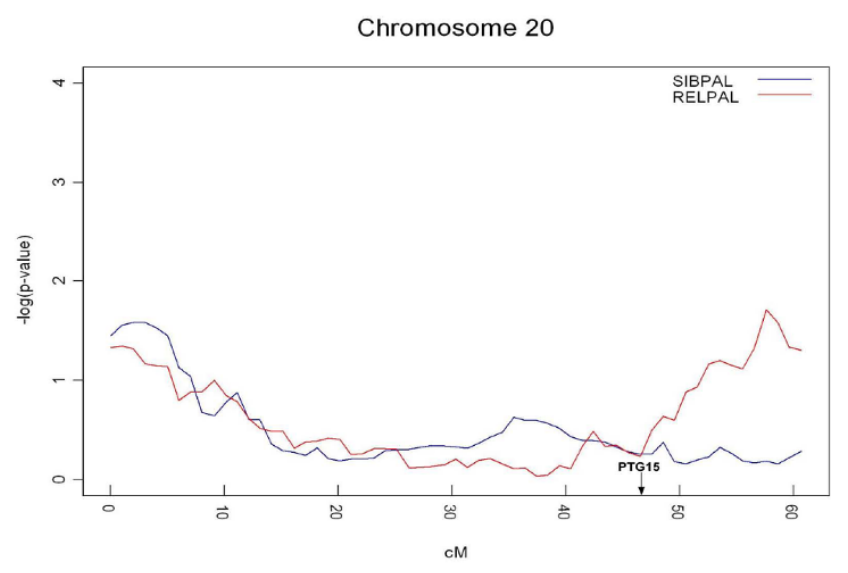

Figure I

Univariate and multivariate linkage analysis results for chromosomes I (A), 3 (B), 12 (C), and 20 (D). - $\log _{10}(p$-value) is plotted against marker location in centimorgans. Univariate results are plotted in red, multivariate results are plotted in blue. Locations of relevant candidate genes also indicated.

near the AGT gene, the multivariate results identified this genomic region more precisely. There were also univariate linkage findings in the vicinity of GNB3 and PTGIS, but not nearly as significant as the multivariate findings (Figures 1C and 1D). These results demonstrate the usefulness of multivariate linkage analysis in mapping complex traits such as HTN, particularly those for which there are highly correlated subphenotypes with large within-family covariance.

\section{Conclusion}

In summary, we observed linkage to chromosomal regions containing candidate genes for HTN. Our multivariate analysis identified more such regions than our univariate analysis. These findings support the use of multivariate linkage analysis when analyzing a number of correlated phenotypes that together predispose to a complex trait like HTN.

\section{List of abbreviations used}

DBP: Diastolic blood pressure; HDL: High-density lipoprotein; HTN: Hypertension; IBD: Identical by descent; LDL: Low-density lipoprotein; SBP: Systolic blood pressure; SNP: Single-nucleotide polymorphism; TG: Triglyceride

\section{Competing interests}

The authors declare that they have no competing interests.

\section{Authors' contributions}

CG-M and CMS conceived of and designed the study and drafted the manuscript. NJM helped conduct the study and draft the manuscript. YS performed the statistical analysis. All authors read and approved the final manuscript. 


\section{Acknowledgements}

The Genetic Analysis Workshops are supported by NIH grant ROI GM031575 from the National Institute of General Medical Sciences. This work is supported by the NCRR Human Genetic Analysis Resource (RR03655), NCRR Multidisciplinary Clinical Research Career Development Programs Grant (KL2RR024990), and NHLBI grant HL-0756. Some of the results of this paper were obtained by using the program package $S$. A.G.E., which is supported by a U.S. Public Health Service Resource Grant (RR03655) from the NCRR. We also thank the participants of GAWI6 group 12 for their helpful comments and suggestions.

This article has been published as part of BMC Proceedings Volume 3 Supplement 7, 2009: Genetic Analysis Workshop 16. The full contents of the supplement are available online at http://www.biomedcentral.com/ $1753-6561 / 3$ ? issue $=$ S7.

\section{References}

I. Eaves L, Neale M and Maes $\mathrm{H}$ : Multivariate multipoint linkage analysis of quantitative trait loci. Behav Genet 1996, 26:5 I 9-525.

2. Fisher SE and DeFries JC: Developmental dyslexia: genetic dissection of a complex cognitive trait. Nat Rev Neurosci 2002, 3:767-780.

3. Allison D, Thiel B, St Jean P, Elston R, Infante $M$ and Schork N: Multiple phenotype modeling in gene-mapping studies of quantitative traits: power advantages. Am J Hum Genet 1998, 63:1190-1201.

4. Amos C, de AM and Zhu D: Comparison of multivariate tests for genetic linkage. Hum Hered 200I, $5 \mathrm{I}$ : I33-144.

5. Marlow AJ, Fisher SE, Francks C, MacPhie IL, Cherny SS, Richardson AJ, Talcott JB, Stein JF, Monaco AP and Cardon LR: Use of multivariate linkage analysis for dissection of a complex cognitive trait. Am J Hum Genet 2003, 72:56I-570.

6. Lifton RP: Molecular genetics of human blood pressure variation. Science 1996, 272:676-680.

7. Jeunemaitre $X$, Soubrier F, Kotelevtsev YV, Lifton RP, Williams CS Charru A, Hunt SC, Hopkins PN, Williams RR, Lalouel JM and Corvol P: Molecular basis of human hypertension: role of angiotensinogen. Cell 1992, 71:169-180.

8. Glenn $C L$, Wang WY, Benjafield $A V$ and Morris BJ: Linkage and association of tumor necrosis factor receptor 2 locus with hypertension, hypercholesterolemia and plasma shed receptor. Hum Mol Genet 2000, 9:1943-1949.

9. Funke-Kaiser H, Reichenberger F, Köpke K, Herrmann SM, Pfeifer J, Orzechowski HD, Zidek W, Paul M and Brand E: Differential binding of transcription factor E2F-2 to the endothelinconverting enzyme-Ib promoter affects blood pressure regulation. Hum Mol Genet 2003, I 2:423-433.

10. Siffert W, Rosskopf D, Siffert G, Busch S, Moritz A, Erbel R, Sharma AM, Ritz E, Wichmann HE, Jakobs KH and Horsthemke B: Association of a human G-protein beta3 subunit variant with hypertension. Nat Genet 1998, 18:45-48.

II. Nakayama T, Soma M, Watanabe Y, Hasimu B, Sato M, Aoi N, Kosuge K, Kanmatsuse K, Kokubun S, Marrow JD and Oates JA: Splicing mutation of the prostacyclin synthase gene in a family associated with hypertension. Biochem Biophys Res Commun 2002, 297: II35-II39.

12. Cui J, Hopper J and Harrap S: Antihypertensive treatments obscure familial contributions to blood pressure variation. Hypertension 2003, 41:207-210.

13. Shete $S$, Jacobs $K$ and Elston R: Adding further power to the Haseman and Elston method for detecting linkage in larger sibships: weighting sums and differences. Hum Hered 2003, 55:79-85.

14. Wang $T$ and Elston RC: Regression-based multivariate linkage analysis with an application to blood pressure and body mass index. Ann Hum Genet 2007, 71:96-106.

15. Wang $T$ and Elston RC: Two-level Haseman-Elston regression for general pedigree data analysis. Genet Epidemiol 2005, 29: $12-22$.

16. Verbeke G and Molenberghs G: The use of score tests for inference on variance components. Biometrics 2003, 59:254-262

17. Morris NJ, Elston R and Stein CM: Calculating asymptotic significance levels of the constrained likelihood ratio test with application to multivariate genetic linkage analysis. Stat Appl Genet Mol Biol 8:39.

18. Cordell H: Sample size requirements to control for stochastic variation in magnitude and location of allele-sharing linkage statistics in affected sibling pairs. Ann Hum Genet 200I, 65:491-502.

19. Ardlie KG, Kruglyak $L$ and Seielstad M: Patterns of linkage disequilibrium in the human genome. Nat Rev Genet 2002, 3:299-309.

20. Clerget-Darpoux F and Elston RC: Are linkage analysis and the collection of family data dead? Prospects for family studies in the age of genome-wide association. Hum Hered 2007 64:91-96.

21. Roeder K, Bacanu SA, Wasserman L and Devlin B: Using linkage genome scans to improve power of association in genome scans. Am J Hum Genet 2006, 78:243-252.
Publish with BioMed Central and every scientist can read your work free of charge

"BioMed Central will be the most significant development for disseminating the results of biomedical research in our lifetime. "

Sir Paul Nurse, Cancer Research UK

Your research papers will be:

- available free of charge to the entire biomedical community

- peer reviewed and published immediately upon acceptance

- cited in PubMed and archived on PubMed Central

- yours - you keep the copyright
BioMedcentral 